\title{
On the Hermitian Positive Definite Solutions of Nonlinear Matrix Equation $X^{s}+A^{*} X^{-t_{1}} A+B^{*} X^{-t_{2}} B=Q$
}

\author{
Aijing Liu' ${ }^{1,2}$ and Guoliang Chen ${ }^{1}$ \\ ${ }^{1}$ Department of Mathematics, East China Normal University, Shanghai 200062, China \\ ${ }^{2}$ School of Mathematical Sciences, Qufu Normal University, Shandong 273165, China
}

Correspondence should be addressed to Guoliang Chen, glchen@math.ecnu.edu.cn

Received 18 April 2011; Accepted 11 July 2011

Academic Editor: Mohammad Younis

Copyright (C) 2011 A. Liu and G. Chen. This is an open access article distributed under the Creative Commons Attribution License, which permits unrestricted use, distribution, and reproduction in any medium, provided the original work is properly cited.

Nonlinear matrix equation $X^{s}+A^{*} X^{-t_{1}} A+B^{*} X^{-t_{2}} B=Q$ has many applications in engineering; control theory; dynamic programming; ladder networks; stochastic filtering; statistics and so forth. In this paper, the Hermitian positive definite solutions of nonlinear matrix equation $X^{s}+A^{*} X^{-t_{1}} A+$ $B^{*} X^{-t_{2}} B=Q$ are considered, where $Q$ is a Hermitian positive definite matrix, $A, B$ are nonsingular complex matrices, $s$ is a positive number, and $0<t_{i} \leq 1, i=1,2$. Necessary and sufficient conditions for the existence of Hermitian positive definite solutions are derived. A sufficient condition for the existence of a unique Hermitian positive definite solution is given. In addition, some necessary conditions and sufficient conditions for the existence of Hermitian positive definite solutions are presented. Finally, an iterative method is proposed to compute the maximal Hermitian positive definite solution, and numerical example is given to show the efficiency of the proposed iterative method.

\section{Introduction}

We consider the nonlinear matrix equation

$$
X^{s}+A^{*} X^{-t_{1}} A+B^{*} X^{-t_{2}} B=Q,
$$

where $Q$ is an $n \times n$ Hermitian positive definite matrix, $A, B$ are $n \times n$ nonsingular complex matrices, $s$ is a positive number, and $0<t_{i} \leq 1, i=1,2$. Here $A^{*}$ stands for the conjugate transpose of the matrix $A$.

Nonlinear matrix equations with the form of (1.1) have many applications in engineering; control theory; dynamic programming; ladder networks; stochastic filtering; statistics and so forth. The solutions of practical interest are their Hermitian positive definite (HPD) 
solutions. The existence of HPD solutions of (1.1) has been investigated in some special cases. Long et al. [1] studied (1.1) when $s=1, t 1=t 2=1$. In addition, there have been many papers considering the Hermitian positive solutions of

$$
X^{s}+A^{*} X^{-t} A=Q
$$

For instance, the authors [2-5] studied (1.2) when $s=1, t=1$. In Hasanov [6, 7], the authors investigated (1.2) when $s=1, t \in(0,1]$. Then Peng et al. [8] proposed iterative methods for the extremal positive definite solutions of (1.2) for $s=1$ with two cases: $0<t \leq 1$ and $t \geq 1$. Cai and Chen $[9,10]$ studied (1.2) with two cases: $s$ and $t$ are positive integers, and $s \geq 1$, $0<t \leq 1$ or $0<s \leq 1, t \geq 1$ respectively.

In this paper, we study the HPD solutions of (1.1). The paper is organized as follows. In Section 2, we derive necessary and sufficient conditions for the existence of HPD solutions of (1.1) and give a sufficient condition for the existence of a unique HPD solution of (1.1). We also present some necessary conditions and sufficient conditions for the existence of HPD solutions of (1.1). Then in Section 3, we propose an iterative method for obtaining the maximal HPD solution of (1.1). We give a numerical example in Section 4 to show the efficiency of the proposed iterative method.

We start with some notations which we use throughout this paper. The symbol $C^{m \times n}$ denotes the set of $m \times n$ complex matrices. We write $D>0(D \geq 0)$ if the matrix $D$ is positive definite(semidefinite). If $D-E$ is positive definite(semidefinite), then we write $D>E(D \geq E)$. We use $\lambda_{1}(D)$ and $\lambda_{n}(D)$ to denote the maximal and minimal eigenvalues of a matrix $D$. We use $\|D\|$ and $\|D\|_{F}$ to denote the spectral and Frobenius norm of a matrix $D$, and we also use $\|b\|$ to denote $l_{2}$-norm of a vector $b$. We use $X_{S}$ and $X_{L}$ to denote the minimal and maximal HPD solution of (1.1), that is, for any HPD solution $X$ of (1.1), then $X_{S} \leq X \leq X_{L}$. The symbol $I$ denotes the $n \times n$ identity matrix. The symbol $\rho(D)$ denotes the spectral radius of $D$. Let $[D, E]=\{X \mid D \leq X \leq E\}$ and $(D, E)=\{X \mid D<X<E\}$. For matrices $D=$ $\left(d_{1}, d_{2}, \ldots, d_{n}\right)=\left(d_{i j}\right)$ and $E, D \otimes E=\left(d_{i j} E\right)$ is a Kronecker product and vec $(D)$ is a vector defined by $\operatorname{vec}(D)=\left(d_{1}^{T}, d_{2}^{T}, \ldots, d_{n}^{T}\right)^{T}$.

\section{Solvability Conditions and Properties of the HPD Solutions}

In this section, we will derive the necessary and sufficient conditions for (1.1) to have an HPD solution and give a sufficient condition for the existence of a unique HPD solution of (1.1). We also will present some necessary conditions and sufficient conditions for the existence of Hermitian positive definite solutions of (1.1).

Lemma 2.1 (see [11]). If $D \geq E>0$ (or $D>E>0$ ), then $D^{p} \geq E^{p}>0$ (or $D^{p}>E^{p}>0$ ) for all $p \in(0,1]$, and $E^{p} \geq D^{p}>0$ (or $E^{p}>D^{p}>0$ ) for all $p \in[-1,0)$.

Lemma 2.2 (see [12]). Let $D$ and $E$ be positive operators on a Hilbert space such that $0<m_{1} I \leq$ $D \leq M_{1} I, 0<m_{2} I \leq E \leq M_{2} I$, and $0<D \leq E$. Then

$$
D^{q} \leq\left(\frac{M_{1}}{m_{1}}\right)^{q-1} E^{q}, \quad D^{q} \leq\left(\frac{M_{2}}{m_{2}}\right)^{q-1} E^{q}
$$

hold for any $q \geq 1$. 
Lemma 2.3 (see [13]). Let $f(x)=x^{t}\left(\zeta-x^{S}\right), \zeta>0, x \geq 0$. Then

(1) $f$ is increasing on $\left[0,((t /(s+t)) \zeta)^{1 / s}\right]$ and decreasing on $\left[((t /(s+t)) \zeta)^{1 / s},+\infty\right)$;

(2) $f_{\max }=f\left(((t /(s+t)) \zeta)^{1 / s}\right)=(s /(s+t))(t /(s+t))^{t / s} \zeta^{(t / s)+1}$.

Lemma 2.4 (see [14]). If $D$ and $E$ are Hermitian matrices of the same order with $E>0$, then DED+ $E^{-1} \geq 2 D$.

Lemma 2.5 (see [15]). If $0<\theta \leq 1$, and $D$ and $E$ are positive definite matrices of the same order with $D, E \geq b I>0$, then $\left\|D^{\theta}-E^{\theta}\right\| \leq \theta b^{\theta-1}\|D-E\|$ and $\left\|D^{-\theta}-E^{-\theta}\right\| \leq \theta b^{-(\theta+1)}\|D-E\|$. Here $\|\cdot\|$ stands for one kind of matrix norm.

Lemma 2.6 (see [5]). Let $D$ and $E$ be two arbitrary compatible matrices. Then $\rho\left(D^{*} E-E^{*} D\right) \leq$ $\rho\left(D^{*} D+E^{*} E\right)$.

Theorem 2.7. Equation (1.1) has an HPD solution if and only if $A, B$ can factor as

$$
A=\left(L^{*} L\right)^{t_{1} / 2 s} N_{1}, \quad B=\left(L^{*} L\right)^{t_{2} / 2 s} N_{2}
$$

where $L$ is a nonsingular matrix and $\left(\begin{array}{c}L Q^{-1 / 2} \\ N_{1} Q^{-1 / 2} \\ N_{2} Q^{-1 / 2}\end{array}\right)$ is column orthonormal.

Proof. If (1.1) has an HPD solution, then $X^{s}>0$. Let $X^{s}=L^{*} L$ be the Cholesky factorization, where $L$ is a nonsingular matrix. Then (1.1) can be rewritten as

$$
\begin{gathered}
Q^{-1 / 2} L^{*} L Q^{-1 / 2}+Q^{-1 / 2} A^{*}\left(L^{*} L\right)^{-t_{1} / 2 s}\left(L^{*} L\right)^{-t_{1} / 2 s} A Q^{-1 / 2} \\
+Q^{-1 / 2} B^{*}\left(L^{*} L\right)^{-t_{2} / 2 s}\left(L^{*} L\right)^{-t_{2} / 2 s} B Q^{-1 / 2}=I
\end{gathered}
$$

Let $N_{1}=\left(L^{*} L\right)^{-t_{1} / 2 s} A, N_{2}=\left(L^{*} L\right)^{-t_{2} / 2 s} B$, then $A=\left(L^{*} L\right)^{t_{1} / 2 s} N_{1}, B=\left(L^{*} L\right)^{t_{2} / 2 s} N_{2}$. Moreover, (2.3) turns into

$$
Q^{-1 / 2} L^{*} L Q^{-1 / 2}+Q^{-1 / 2} N_{1}^{*} N_{1} Q^{-1 / 2}+Q^{-1 / 2} N_{2}^{*} N_{2} Q^{-1 / 2}=I,
$$

that is,

$$
\left(\begin{array}{c}
L Q^{-1 / 2} \\
N_{1} Q^{-1 / 2} \\
N_{2} Q^{-1 / 2}
\end{array}\right)^{*}\left(\begin{array}{c}
L Q^{-1 / 2} \\
N_{1} Q^{-1 / 2} \\
N_{2} Q^{-1 / 2}
\end{array}\right)=I
$$

which means that $\left(\begin{array}{c}L Q^{-1 / 2} \\ N_{1} Q^{-1 / 2} \\ N_{2} Q^{-1 / 2}\end{array}\right)$ is column orthonormal. 
Conversely, if $A, B$ have the decompositions as (2.2), let $X=\left(L^{*} L\right)^{1 / s}$, then $X$ is an HPD matrix, and it follows from (2.2) and (2.4) that

$$
\begin{aligned}
X^{S}+A^{*} X^{-t_{1}} A+B^{*} X^{-t_{2}} B= & L^{*} L+N_{1}^{*} N_{1}+N_{2}^{*} N_{2} \\
= & Q^{1 / 2}\left(Q^{-1 / 2} L^{*} L Q^{-1 / 2}+Q^{-1 / 2} N_{1}^{*} N_{1} Q^{-1 / 2}\right. \\
& \left.\quad+Q^{-1 / 2} N_{2}^{*} N_{2} Q^{-1 / 2}\right) Q^{1 / 2} \\
& =Q .
\end{aligned}
$$

Hence (1.1) has an HPD solution.

Theorem 2.8. Equation (1.1) has an HPD solution if and only if there exist a unitary matrix $V \in$ $C^{n \times n}$, a column-orthonormal matrix $U=\left(\begin{array}{l}U_{1} \\ U_{2}\end{array}\right) \in C^{2 n \times n}$ (in which $U_{1}, U_{2} \in C^{n \times n}$ ), and diagonal matrices $C>0$ and $S \geq 0$ with $C^{2}+S^{2}=I$ such that

$$
\begin{aligned}
& A=\left(Q^{1 / 2} V^{*} C^{2} V Q^{1 / 2}\right)^{t_{1} / 2 s} U_{1} S V Q^{1 / 2}, \\
& B=\left(Q^{1 / 2} V^{*} C^{2} V Q^{1 / 2}\right)^{t_{2} / 2 s} U_{2} S V Q^{1 / 2} .
\end{aligned}
$$

Proof. If (1.1) has an HPD solution, we have by Theorem 2.7 that the matrix $\left(\begin{array}{c}L Q^{-1 / 2} \\ N_{1} Q^{-1 / 2} \\ N_{2} Q^{-1 / 2}\end{array}\right)$ is column orthonormal. According to the CS decomposition theorem (Theorem 3.8 in [16]), there exist unitary matrices $P=\left(\begin{array}{cc}P_{1} & 0 \\ 0 & P_{2}\end{array}\right) \in \mathrm{C}^{3 n \times 3 n}$ (in which $P_{1} \in \mathrm{C}^{n \times n}, P_{2} \in \mathrm{C}^{2 n \times 2 n}$ ), $V \in \mathrm{C}^{n \times n}$, such that

$$
\left(\begin{array}{cc}
P_{1} & 0 \\
0 & P_{2}
\end{array}\right)\left(\begin{array}{c}
\frac{L Q^{-1 / 2}}{N_{1} Q^{-1 / 2}} \\
N_{2} Q^{-1 / 2}
\end{array}\right) V^{*}=\left(\begin{array}{c}
\frac{C}{S} \\
0
\end{array}\right),
$$

where $C=\operatorname{diag}\left(\cos \theta_{1}, \ldots, \cos \theta_{n}\right), S=\operatorname{diag}\left(\sin \theta_{1}, \ldots, \sin \theta_{n}\right)$, and $0 \leq \theta_{1} \leq \cdots \leq \theta_{n} \leq \pi / 2$. Thus the diagonal matrices $C, S \geq 0$ and $C^{2}+S^{2}=I$. Furthermore, noting that $L$ is nonsingular, by $(2.8)$, we have

$$
\begin{gathered}
C=P_{1} L Q^{-1 / 2} V^{*}>0, \\
P_{2}\left(\begin{array}{c}
N_{1} \\
N_{2}
\end{array}\right) Q^{-1 / 2} V^{*}=\left(\begin{array}{l}
S \\
0
\end{array}\right) .
\end{gathered}
$$


Equation (2.10) is equivalent to $\left(\begin{array}{l}N_{1} \\ N_{2}\end{array}\right)=P_{2}^{*}\left(\begin{array}{l}S \\ 0\end{array}\right) V Q^{1 / 2}$. Let $P_{2}^{*}$ be partitioned as $P_{2}^{*}=$ $\left(\begin{array}{ll}U_{1} & U_{3} \\ U_{2} & U_{4}\end{array}\right)$, in which $U_{i} \in \mathrm{C}^{n \times n}, i=1,2,3,4$, then we have

$$
\left(\begin{array}{l}
N_{1} \\
N_{2}
\end{array}\right)=\left(\begin{array}{ll}
U_{1} & U_{3} \\
U_{2} & U_{4}
\end{array}\right)\left(\begin{array}{l}
S \\
0
\end{array}\right) V Q^{1 / 2}=\left(\begin{array}{l}
U_{1} S V Q^{1 / 2} \\
U_{2} S V Q^{1 / 2}
\end{array}\right)
$$

from which it follows that $N_{1}=U_{1} S V Q^{1 / 2}, N_{2}=U_{2} S V Q^{1 / 2}$. By (2.9), we have $L=$ $P_{1}^{*} C V Q^{1 / 2}$. Then by (2.2), we have

$$
\begin{aligned}
& A=\left(L^{*} L\right)^{t_{1} / 2 s} N_{1}=\left(Q^{1 / 2} V^{*} C^{2} V Q^{1 / 2}\right)^{t_{1} / 2 s} U_{1} S V Q^{1 / 2}, \\
& B=\left(L^{*} L\right)^{t_{2} / 2 s} N_{2}=\left(Q^{1 / 2} V^{*} C^{2} V Q^{1 / 2}\right)^{t_{2} / 2 s} U_{2} S V Q^{1 / 2} .
\end{aligned}
$$

Conversely, assume that $A, B$ have the decomposition (2.7). Let $X=\left(Q^{1 / 2} V^{*} C^{2} V Q^{1 / 2}\right)^{1 / s}$, which is an HPD matrix. Then it is easy to verify that $X$ is an HPD solution of (1.1).

Theorem 2.9. If (1.1) has an HPD solution $X$, then $X \in(\bar{M}, \bar{N})$, where

$$
\begin{gathered}
\bar{M}=\frac{1}{2}\left(\left(\frac{\mu_{1}}{v_{1}}\right)^{\left(1-t_{1}\right) / t_{1}}\left(A Q^{-1} A^{*}\right)^{1 / t_{1}}+\left(\frac{\mu_{2}}{v_{2}}\right)^{\left(1-t_{2}\right) / t_{2}}\left(B Q^{-1} B^{*}\right)^{1 / t_{2}}\right), \\
\bar{N}=\left(Q-A^{*} Q^{-t_{1} / s} A-B^{*} Q^{-t_{2} / s} B\right)^{1 / s},
\end{gathered}
$$

in which $\mu_{1}$ and $v_{1}$ are the minimal and maximal eigenvalues of $A Q^{-1} A^{*}$ respectively, $\mu_{2}$ and $v_{2}$ are the minimal and maximal eigenvalues of $B Q^{-1} B^{*}$, respectively.

Proof. Let $X$ be an HPD solution of (1.1), then it follows from $0<X^{s}<Q$ and Lemma 2.1 that $X^{-t_{i}}>Q^{-t_{i} / s}, i=1,2$. Hence

$$
X^{s}=Q-A^{*} X^{-t_{1}} A-B^{*} X^{-t_{2}} B<Q-A^{*} Q^{-t_{1} / s} A-B^{*} Q^{-t_{2} / s} B
$$

Thus we have

$$
X<\left(Q-A^{*} Q^{-t_{1} / s} A-B^{*} Q^{-t_{2} / s} B\right)^{1 / s}=\bar{N}
$$

On the other hand, from $A^{*} X^{-t_{1}} A<Q$, it follows that

$$
\begin{gathered}
Q^{-1 / 2} A^{*} X^{-t_{1} / 2} X^{-t_{1} / 2} A Q^{-1 / 2}<I, \\
X^{-t_{1} / 2} A Q^{-1} A^{*} X^{-t_{1} / 2}<I, \\
A Q^{-1} A^{*}<X^{t_{1}} .
\end{gathered}
$$


Let $\mu_{1}$ and $\nu_{1}$ be the minimal and maximal eigenvalues of $A Q^{-1} A^{*}$, respectively. Since $1 / t_{1} \geq$ 1 , and $\mu_{1} I \leq A Q^{-1} A^{*} \leq v_{1} I$, by Lemma 2.2, we get $\left(\mu_{1} / v_{1}\right)^{\left(1-t_{1}\right) / t_{1}}\left(A Q^{-1} A^{*}\right)^{1 / t_{1}}<X$.

Similarly, we have $\left(\mu_{2} / v_{2}\right)^{\left(1-t_{2}\right) / t_{2}}\left(B Q^{-1} B^{*}\right)^{1 / t_{2}}<X$, in which $\mu_{2}$ and $v_{2}$ are the minimal and maximal eigenvalues of $B Q^{-1} B^{*}$, respectively.

$\bar{M}$.

Hence we have $X>1 / 2\left(\left(\mu_{1} / v_{1}\right)^{\left(1-t_{1}\right) / t_{1}}\left(A Q^{-1} A^{*}\right)^{1 / t_{1}}+\left(\mu_{2} / v_{2}\right)^{\left(1-t_{2}\right) / t_{2}}\left(B Q^{-1} B^{*}\right)^{1 / t_{2}}\right)=$

Theorem 2.10. If $A^{*} X^{-t_{1}} A+B^{*} X^{-t_{2}} B \leq Q-\bar{M}^{s}$ for all $X \in\left[\bar{M}, Q^{1 / s}\right]$, and

$$
p=\frac{1}{S}\left(t_{1} \lambda_{n}^{-\left(s+t_{1}\right)}(\bar{M})\|A\|_{F}^{2}+t_{2} \lambda_{n}^{-\left(s+t_{2}\right)}(\bar{M})\|B\|_{F}^{2}\right)<1,
$$

where $\bar{M}$ is defined by (2.13), then (1.1) has a unique HPD solution.

Proof. By the definition of $\bar{M}$, we have $\bar{M}>0$. Hence $\lambda_{n}(\bar{M})>0$.

We consider the map $F(X)=\left(Q-A^{*} X^{-t_{1}} A-B^{*} X^{-t_{2}} B\right)^{1 / s}$ and let $X \in \Omega=\{X \mid \bar{M} \leq$ $\left.X \leq Q^{1 / s}\right\}$. Obviously, $\Omega$ is a convex, closed, and bounded set and $F(X)$ is continuous on $\Omega$.

By the hypothesis of the theorem, we have

$$
Q^{1 / s} \geq\left(Q-A^{*} X^{-t_{1}} A-B^{*} X^{-t_{2}} B\right)^{1 / s} \geq\left(Q-Q+\bar{M}^{s}\right)^{1 / s}=\bar{M}
$$

that is, $\bar{M} \leq F(X) \leq Q^{1 / s}$. Hence $F(\Omega) \subseteq \Omega$.

For arbitrary $X, Y \in \Omega$, we have

$$
A^{*} X^{-t_{1}} A+B^{*} X^{-t_{2}} B \leq Q-\bar{M}^{s}, \quad A^{*} Y^{-t_{1}} A+B^{*} Y^{-t_{2}} B \leq Q-\bar{M}^{s}
$$

Hence

$$
\begin{aligned}
& F(X)=\left(Q-A^{*} X^{-t_{1}} A-B^{*} X^{-t_{2}} B\right)^{1 / s} \geq\left(Q-Q+\bar{M}^{s}\right)^{1 / s}=\bar{M} \geq \lambda_{n}(\bar{M}) I \\
& F(Y)=\left(Q-A^{*} Y^{-t_{1}} A-B^{*} Y^{-t_{2}} B\right)^{1 / s} \geq\left(Q-Q+\bar{M}^{s}\right)^{1 / s}=\bar{M} \geq \lambda_{n}(\bar{M}) I
\end{aligned}
$$

From (2.20), it follows that

$$
\begin{aligned}
\left\|F(X)^{s}-F(Y)^{s}\right\|_{F} & =\left\|\sum_{i=0}^{s-1} F(X)^{i}(F(X)-F(Y)) F(Y)^{s-1-i}\right\|_{F} \\
& =\left\|\operatorname{vec}\left[\sum_{i=0}^{s-1} F(X)^{i}(F(X)-F(Y)) F(Y)^{s-1-i}\right]\right\| \\
& =\left\|\sum_{i=0}^{s-1} \operatorname{vec}\left[F(X)^{i}(F(X)-F(Y)) F(Y)^{s-1-i}\right]\right\| \\
& =\left\|\sum_{i=0}^{s-1}\left(F(Y)^{s-1-i} \otimes F(X)^{i}\right) \operatorname{vec}(F(X)-F(Y))\right\|
\end{aligned}
$$


Mathematical Problems in Engineering

$$
\begin{aligned}
& \geq \sum_{i=0}^{s-1} \lambda_{n}^{s-1}(\bar{M})\|\operatorname{vec}(F(X)-F(Y))\| \\
& =s \lambda_{n}^{s-1}(\bar{M})\|F(X)-F(Y)\|_{F} .
\end{aligned}
$$

According to the definition of the map $F$, we have

$$
\begin{aligned}
F(X)^{S}-F(Y)^{S} & =\left(Q-A^{*} X^{-t_{1}} A-B^{*} X^{-t_{2}} B\right)-\left(Q-A^{*} Y^{-t_{1}} A-B^{*} Y^{-t_{2}} B\right) \\
& =A^{*}\left(Y^{-t_{1}}-X^{-t_{1}}\right) A+B^{*}\left(Y^{-t_{2}}-X^{-t_{2}}\right) B .
\end{aligned}
$$

Combining (2.21) and (2.22), we have by Lemma 2.5 that

$$
\begin{aligned}
\|F(X)-F(Y)\|_{F} & \leq \frac{1}{s \lambda_{n}^{s-1}(\bar{M})}\left\|F(X)^{s}-F(Y)^{s}\right\|_{F} \\
& =\frac{1}{s \lambda_{n}^{s-1}(\bar{M})}\left\|A^{*}\left(Y^{-t_{1}}-X^{-t_{1}}\right) A+B^{*}\left(Y^{-t_{2}}-X^{-t_{2}}\right) B\right\|_{F} \\
& \leq \frac{1}{s \lambda_{n}^{s-1}(\bar{M})}\left(\|A\|_{F}^{2}\left\|Y^{-t_{1}}-X^{-t_{1}}\right\|_{F}+\|B\|_{F}^{2}\left\|Y^{-t_{2}}-X^{-t_{2}}\right\|_{F}\right) \\
& \leq \frac{1}{s \lambda_{n}^{s-1}(\bar{M})}\left(t_{1} \lambda_{n}^{-\left(t_{1}+1\right)}(\bar{M})\|A\|_{F}^{2}+t_{2} \lambda_{n}^{-\left(t_{2}+1\right)}(\bar{M})\|B\|_{F}^{2}\right)\|Y-X\|_{F} \\
& =\frac{1}{S}\left(t_{1} \lambda_{n}^{-\left(s+t_{1}\right)}(\bar{M})\|A\|_{F}^{2}+t_{2} \lambda_{n}^{-\left(s+t_{2}\right)}(\bar{M})\|B\|_{F}^{2}\right)\|X-Y\|_{F} \\
& =p\|X-Y\|_{F} .
\end{aligned}
$$

Since $p<1$, we know that the map $F(X)$ is a contraction map in $\Omega$. By Banach fixed point theorem, the map $F(X)$ has a unique fixed point in $\Omega$ and this shows that (1.1) has a unique HPD solution in $\left[\bar{M}, Q^{1 / s}\right]$.

Theorem 2.11. If (1.1) has an HPD solution $X$, then

$$
\lambda_{n}\left(Q^{-1 / 2} A^{*} Q^{-t_{1} / s} A Q^{-1 / 2}+Q^{-1 / 2} B^{*} Q^{-t_{2} / s} B Q^{-1 / 2}\right) \leq\left(\frac{t}{s+t}\right)^{t / s} \frac{s}{s+t}, \quad X \leq \widehat{\alpha} Q^{1 / s},
$$

where $t=\min \left\{t_{1}, t_{2}\right\}$, and $\widehat{\alpha}$ is a solution of the equation

$$
y^{t}\left(1-y^{s}\right)=\lambda_{n}\left(Q^{-1 / 2} A^{*} Q^{-t_{1} / s} A Q^{-1 / 2}+Q^{-1 / 2} B^{*} Q^{-t_{2} / s} B Q^{-1 / 2}\right)
$$

in $\left[(t /(s+t))^{1 / s}, 1\right]$ 
Proof. Consider the sequence defined as follows:

$$
\alpha_{0}=1, \quad \alpha_{k+1}=\left(1-\frac{\lambda_{n}\left(Q^{-1 / 2} A^{*} Q^{-t_{1} / s} A Q^{-1 / 2}+Q^{-1 / 2} B^{*} Q^{-t_{2} / s} B Q^{-1 / 2}\right)}{\alpha_{k}^{t}}\right)^{1 / s},
$$

Let $X$ be an HPD solution of (1.1), then

$$
X=\left(Q-A^{*} X^{-t_{1}} A-B^{*} X^{-t_{2}} B\right)^{1 / s}<Q^{1 / s}=\alpha_{0} Q^{1 / s} .
$$

Assuming that $X<\alpha_{k} Q^{1 / s}$, then by Lemma 2.1, we have

$$
\begin{aligned}
X^{s} & =Q-A^{*} X^{-t_{1}} A-B^{*} X^{-t_{2}} B \\
& <Q-A^{*}\left(\alpha_{k} Q^{1 / s}\right)^{-t_{1}} A-B^{*}\left(\alpha_{k} Q^{1 / s}\right)^{-t_{2}} B \\
& <Q-\frac{A^{*} Q^{-t_{1} / s} A+B^{*} Q^{-t_{2} / s} B}{\alpha_{k}^{t}} \\
& =Q^{1 / 2}\left(I-\frac{Q^{-1 / 2} A^{*} Q^{-t_{1} / s} A Q^{-1 / 2}+Q^{-1 / 2} B^{*} Q^{-t_{2} / s} B Q^{-1 / 2}}{\alpha_{k}^{t}}\right) Q^{1 / 2} \\
& \leq Q^{1 / 2}\left(1-\frac{\lambda_{n}\left(Q^{-1 / 2} A^{*} Q^{-t_{1} / s} A Q^{-1 / 2}+Q^{-1 / 2} B^{*} Q^{-t_{2} / s} B Q^{-1 / 2}\right)}{\alpha_{k}^{t}}\right) Q^{1 / 2} \\
& =\alpha_{k+1}^{s} Q .
\end{aligned}
$$

Therefore $X<\alpha_{k+1} Q^{1 / s}$. Then by the principle of induction, we get $X<\alpha_{k} Q^{1 / s}, k=0,1,2, \ldots$ Noting that the sequence $\alpha_{k}$ is monotonically decreasing and positive, hence $\alpha_{k}$ is convergent. Let $\lim _{k \rightarrow \infty} \alpha_{k}=\widehat{\alpha}$, then $\widehat{\alpha}=\left(1-\lambda_{n}\left(Q^{-1 / 2} A^{*} Q^{-t_{1} / s} A Q^{-1 / 2}+Q^{-1 / 2} B^{*} Q^{-t_{2} / s} B Q^{-1 / 2}\right) /\right.$ $\left.\widehat{\alpha}^{t}\right)^{1 / s}$, that is, $\widehat{\alpha}$ is a solution of the equation $y^{t}\left(1-y^{s}\right)=\lambda_{n}\left(Q^{-1 / 2} A^{*} Q^{-t_{1} / s} A Q^{-1 / 2}+\right.$ $\left.Q^{-1 / 2} B^{*} Q^{-t_{2} / s} B Q^{-1 / 2}\right)$.

Consider the function $f(y)=y^{t}\left(1-y^{s}\right)$, since

$$
\max _{y \in[0,1]}=f\left(\left(\frac{t}{s+t}\right)^{1 / s}\right)=\left(\frac{t}{s+t}\right)^{t / s} \frac{s}{s+t},
$$

from which it follows that $\lambda_{n}\left(Q^{-1 / 2} A^{*} Q^{-t_{1} / s} A Q^{-1 / 2}+Q^{-1 / 2} B^{*} Q^{-t_{2} / s} B Q^{-1 / 2}\right) \leq(t /(s+t))^{t / s}(s /$ $(s+t))$. 
Next we will prove that $\widehat{\alpha} \in\left[(t /(s+t))^{1 / s}, 1\right]$. Obviously, $\widehat{\alpha} \leq 1$. On the other hand, for the sequence $\alpha_{k}$, since $\alpha_{0}=1>(t /(s+t))^{1 / s}$, we may assume that $\alpha_{k}>(t /(s+t))^{1 / s}$ without loss of generality. Then

$$
\begin{aligned}
\alpha_{k+1} & =\left(1-\frac{\lambda_{n}\left(Q^{-1 / 2} A^{*} Q^{-t_{1} / s} A Q^{-1 / 2}+Q^{-1 / 2} B^{*} Q^{-t_{2} / s} B Q^{-1 / 2}\right)}{\alpha_{k}^{t}}\right)^{1 / s} \\
& \geq\left(1-\frac{1}{\alpha_{k}^{t}}\left(\frac{t}{s+t}\right)^{t / s} \frac{s}{s+t}\right)^{1 / s} \\
& >\left(1-\frac{1}{(t /(s+t))^{t / s}}\left(\frac{t}{s+t}\right)^{t / s} \frac{s}{s+t}\right)^{1 / s} \\
& =\left(\frac{t}{s+t}\right)^{1 / s} \cdot
\end{aligned}
$$

Hence $\alpha_{k}>(t /(s+t))^{1 / s}, k=0,1,2, \ldots$. So $\widehat{\alpha}=\lim _{k \rightarrow \infty} \alpha_{k} \geq(t /(s+t))^{1 / s}$.

Consequently, we have $\widehat{\alpha} \in\left[(t /(s+t))^{1 / s}, 1\right]$.

This completes the proof.

Theorem 2.12. If (1.1) has an HPD solution, then

$$
\begin{aligned}
& (\rho(A))^{2} \leq \frac{s}{s+t_{1}}\left(\frac{t_{1}}{s+t_{1}}\right)^{t_{1} / s}(\rho(Q))^{\left(t_{1} / s\right)+1} \\
& (\rho(B))^{2} \leq \frac{s}{s+t_{2}}\left(\frac{t_{2}}{s+t_{2}}\right)^{t_{2} / s}(\rho(Q))^{\left(t_{2} / s\right)+1}
\end{aligned}
$$

Proof. For any eigenvalue $\lambda(A)$ of $A$, let $x$ be a corresponding eigenvector. Multiplying left side of (1.1) by $x^{*}$ and right side by $x$, we have

$$
x^{*} X^{s} x+x^{*} A^{*} X^{-t_{1}} A x+x^{*} B^{*} X^{-t_{2}} B x=x^{*} Q x,
$$

which yields

$$
x^{*} X^{s} x+|\lambda(A)|^{2} x^{*} X^{-t_{1}} x+x^{*} B^{*} X^{-t_{2}} B x=x^{*} Q x .
$$

Since $X>0$, there exists an unitary matrix $U$ such that $X=U^{*} \Lambda U$, where $\Lambda=\operatorname{diag}\left(\eta_{1}, \ldots\right.$, $\left.\eta_{n}\right)>0$. Then (2.34) turns into the following form:

$$
x^{*} U^{*} \Lambda^{s} U x+|\lambda(A)|^{2} x^{*} U^{*} \Lambda^{-t_{1}} U x \leq x^{*} Q x .
$$

Let $y=\left(y_{1}, y_{2}, \ldots, y_{n}\right)^{T}=U x$, then $(2.35)$ reduces to

$$
y^{*} \Lambda^{s} y+|\lambda(A)|^{2} y^{*} \Lambda^{-t_{1}} y \leq y^{*} U Q U^{*} y,
$$


from which we obtain

$$
|\lambda(A)|^{2} \leq \frac{y^{*}\left(U Q U^{*}-\Lambda^{s}\right) y}{y^{*} \Lambda^{-t_{1}} y} \leq \frac{y^{*}\left(\lambda_{1}(Q) I-\Lambda^{s}\right) y}{y^{*} \Lambda^{-t_{1}} y}=\frac{\sum_{i=1}^{n} y_{i}^{2}\left(\lambda_{1}(Q)-\eta_{i}^{s}\right)}{\sum_{i=1}^{n} y_{i}^{2} \eta_{i}^{-t_{1}}} .
$$

Form Lemma 2.3, we know that

$$
\eta_{i}^{t_{1}}\left(\lambda_{1}(Q)-\eta_{i}^{s}\right) \leq \frac{s}{s+t_{1}}\left(\frac{t_{1}}{s+t_{1}}\right)^{t_{1} / s} \lambda_{1}^{\left(t_{1} / s\right)+1}(Q),
$$

that is,

$$
\left(\lambda_{1}(Q)-\eta_{i}^{s}\right) \leq \frac{s}{s+t_{1}}\left(\frac{t_{1}}{s+t_{1}}\right)^{t_{1} / s} \lambda_{1}^{\left(t_{1} / s\right)+1}(Q) \eta_{i}^{-t_{1}}
$$

Noting that $y \neq 0$, we get

$$
\sum_{i=1}^{n} y_{i}^{2}\left(\lambda_{1}(Q)-\eta_{i}^{s}\right) \leq \frac{s}{s+t_{1}}\left(\frac{t_{1}}{s+t_{1}}\right)^{t_{1} / s} \lambda_{1}^{\left(t_{1} / s\right)+1}(Q) \sum_{i=1}^{n} y_{i}^{2} \eta_{i}^{-t_{1}}
$$

Consequently,

$$
|\lambda(A)|^{2} \leq \frac{\sum_{i=1}^{n} y_{i}^{2}\left(\lambda_{1}(Q)-\eta_{i}^{s}\right)}{\sum_{i=1}^{n} y_{i}^{2} \eta_{i}^{-t_{1}}} \leq \frac{s}{s+t_{1}}\left(\frac{t_{1}}{s+t_{1}}\right)^{t_{1} / s} \lambda_{1}^{\left(t_{1} / s\right)+1}(Q) .
$$

Then $(\rho(A))^{2} \leq\left(s /\left(s+t_{1}\right)\right)\left(t_{1} /\left(s+t_{1}\right)\right)^{t_{1} / s} \lambda_{1}^{\left(t_{1} / s\right)+1}(Q)$.

Since $Q>0$, clearly denote $\lambda_{1}(Q)=\rho(Q)$, and the last inequality implies directly (2.31).

The proof of (2.32) is similar to that of (2.31), thus it is omitted here.

Theorem 2.13. If $Q \leq I$ and (1.1) has an HPD solution, then

$$
\begin{array}{ll}
\rho\left(A^{s / t_{1}}+\left(A^{*}\right)^{s / t_{1}}\right) \leq \rho(Q), & \rho\left(A^{s / t_{1}}-\left(A^{*}\right)^{s / t_{1}}\right) \leq \rho(Q), \\
\rho\left(B^{s / t_{2}}+\left(B^{*}\right)^{s / t_{2}}\right) \leq \rho(Q), & \rho\left(B^{s / t_{2}}-\left(B^{*}\right)^{s / t_{2}}\right) \leq \rho(Q) .
\end{array}
$$

Proof. If (1.1) has an HPD solution, we have by Theorem 2.7 that

$$
A=\left(L^{*} L\right)^{t_{1} / 2 s} N_{1}, \quad B=\left(L^{*} L\right)^{t_{2} / 2 s} N_{2}
$$

and the matrix $\left(\begin{array}{c}L Q^{-1 / 2} \\ N_{1} Q^{-1 / 2} \\ N_{2} Q^{-1 / 2}\end{array}\right)$ is column orthonormal. From which we have

$$
L^{*} L+N_{1}^{*} N_{1}+N_{2}^{*} N_{2}=Q .
$$


Hence,

$$
\begin{aligned}
Q-\left(A^{s / t_{1}}+\left(A^{*}\right)^{s / t_{1}}\right)= & L^{*} L+N_{1}^{*} N_{1}+N_{2}^{*} N_{2}-\left(L^{*} L\right)^{1 / 2} N_{1}^{s / t_{1}}-\left(N_{1}^{*}\right)^{s / t_{1}}\left(L^{*} L\right)^{1 / 2} \\
= & \left(\left(L^{*} L\right)^{1 / 2}-N_{1}^{s / t_{1}}\right)^{*}\left(\left(L^{*} L\right)^{1 / 2}-N_{1}^{s / t_{1}}\right) \\
& +N_{2}^{*} N_{2}+\left(N_{1}^{*} N_{1}-\left(N_{1}^{*}\right)^{s / t_{1}} N_{1}^{s / t_{1}}\right) \\
\geq & 0 .
\end{aligned}
$$

Similarly, we have $Q+\left(A^{s / t_{1}}+\left(A^{*}\right)^{s / t_{1}}\right) \geq 0$.

Thus, $-Q \leq\left(A^{s / t_{1}}+\left(A^{*}\right)^{s / t_{1}}\right) \leq Q$. Hence $\rho\left(A^{s / t_{1}}+\left(A^{*}\right)^{s / t_{1}}\right) \leq \rho(Q)$.

On the other hand, by Lemma 2.6 and (2.2), we get

$$
\begin{aligned}
\rho\left(A^{s / t_{1}}-\left(A^{*}\right)^{S / t_{1}}\right) & =\rho\left(\left(L^{*} L\right)^{1 / 2} N_{1}^{S / t_{1}}-\left(N_{1}^{*}\right)^{s / t_{1}}\left(L^{*} L\right)^{1 / 2}\right) \\
& \leq \rho\left(L^{*} L+\left(N_{1}^{*}\right)^{s / t_{1}} N_{1}^{s / t_{1}}\right) \\
& \leq \rho\left(L^{*} L+N_{1}^{*} N_{1}\right) \\
& \leq \rho(Q) .
\end{aligned}
$$

The proof of (2.43) is similar to that of (2.42).

$$
\text { If } t_{1}=t_{2} \text {, we denote } t=t_{1}=t_{2} \text {. Then (1.1) turns into }
$$

$$
X^{s}+A^{*} X^{-t} A+B^{*} X^{-t} B=Q
$$

Consider the following equations:

$$
\begin{aligned}
& x^{s+t}-\lambda_{n}(Q) x^{t}+\lambda_{1}\left(A^{*} A\right)+\lambda_{1}\left(B^{*} B\right)=0, \\
& x^{s+t}-\lambda_{1}(Q) x^{t}+\lambda_{n}\left(A^{*} A\right)+\lambda_{n}\left(B^{*} B\right)=0 .
\end{aligned}
$$

We assume that $A, B$, and $Q$ satisfy

$$
\lambda_{1}\left(A^{*} A\right)+\lambda_{1}\left(B^{*} B\right)<\frac{s}{s+t} \xi_{*}^{t} \lambda_{n}(Q)
$$

where $\xi_{*}=\left((t /(s+t)) \lambda_{n}(Q)\right)^{1 / s}$. By (2.51) and Lemma 2.3, we know that (2.49) has two positive real roots $\alpha_{2}<\beta_{1}$. We also get that (2.50) has two positive real roots $\alpha_{1}<\beta_{2}$. It is easy to prove that

$$
0<\alpha_{1} \leq \alpha_{2}<\xi_{*}<\beta_{1} \leq \beta_{2}<\lambda_{1}^{1 / s}(Q)
$$


We define matrix sets as follows:

$$
\begin{aligned}
& \varphi_{1}=\left\{X=X^{*} \mid 0<X<\alpha_{1} I\right\}, \\
& \varphi_{2}=\left\{X=X^{*} \mid \alpha_{1} I \leq X \leq \alpha_{2} I\right\}, \\
& \varphi_{3}=\left\{X=X^{*} \mid \alpha_{2} I<X<\beta_{1} I\right\}, \\
& \varphi_{4}=\left\{X=X^{*} \mid \beta_{1} I \leq X \leq \beta_{2} I\right\}, \\
& \varphi_{5}=\left\{X=X^{*} \mid \beta_{2} I<X<\lambda_{1}^{1 / s}(Q) I\right\} .
\end{aligned}
$$

Theorem 2.14. Suppose that $A, B$, and $Q$ satisfy (2.51), that is,

$$
\lambda_{1}\left(A^{*} A\right)+\lambda_{1}\left(B^{*} B\right)<\frac{s}{s+t}\left(\frac{t}{s+t}\right)^{t / s} \lambda_{n}^{(t / s)+1}(Q)
$$

Then

(i) Equation (2.48) has a unique HPD solution in $\varphi_{4}$;

(ii) Equation (2.48) has no HPD solution in $\varphi_{1}, \varphi_{3}, \varphi_{5}$.

Proof. Consider the map $G(X)=\left(Q-A^{*} X^{-t} A-B^{*} X^{-t} B\right)^{1 / s}$, which is continuous on $\varphi_{4}$. Obviously, $\varphi_{4}$ is a convex, closed, and bounded set. If $X \in \varphi_{4}$,

$$
\begin{aligned}
\lambda_{1}^{s}(G(X))=\lambda_{1}\left(G(X)^{s}\right) & =\lambda_{1}\left(Q-A^{*} X^{-t} A-B^{*} X^{-t} B\right) \\
& \leq \lambda_{1}(Q)-\frac{\lambda_{n}\left(A^{*} A\right)+\lambda_{n}\left(B^{*} B\right)}{\lambda_{1}^{t}(X)} \\
& \leq \lambda_{1}(Q)-\frac{\lambda_{n}\left(A^{*} A\right)+\lambda_{n}\left(B^{*} B\right)}{\beta_{2}^{t}} \\
& =\beta_{2}^{s} .
\end{aligned}
$$

Hence, we have $\lambda_{1}(G(X))<\beta_{2}$. One has

$$
\begin{aligned}
\lambda_{n}^{s}(G(X))=\lambda_{n}\left(G(X)^{s}\right) & =\lambda_{n}\left(Q-A^{*} X^{-t} A-B^{*} X^{-t} B\right) \\
& \geq \lambda_{n}(Q)-\frac{\lambda_{1}\left(A^{*} A\right)+\lambda_{1}\left(B^{*} B\right)}{\lambda_{n}^{t}(X)} \\
& \geq \lambda_{n}(Q)-\frac{\lambda_{1}\left(A^{*} A\right)+\lambda_{1}\left(B^{*} B\right)}{\beta_{1}^{t}} \\
& =\beta_{1}^{s} .
\end{aligned}
$$

Hence, we have $\lambda_{n}(G(X))<\beta_{1}$.

Thus, $G(X)$ maps $\varphi_{4}$ into itself. 
For arbitrary $X, Y \in \varphi_{4}$, similar to (2.21) and (2.22), we have

$$
\begin{gathered}
\left\|G(X)^{s}-G(Y)^{s}\right\|_{F} \geq s \beta_{1}^{s-1}\|G(X)-G(Y)\|_{F^{\prime}} \\
G(X)^{s}-G(Y)^{s}=A^{*}\left(Y^{-t}-X^{-t}\right) A+B^{*}\left(Y^{-t}-X^{-t}\right) B .
\end{gathered}
$$

Combining (2.57), we have by Lemma 2.5 and (2.49)

$$
\begin{aligned}
\|G(X)-G(Y)\|_{F} & \leq \frac{1}{s \beta_{1}^{s-1}}\left\|G(X)^{s}-G(Y)^{s}\right\|_{F} \\
& =\frac{1}{s \beta_{1}^{s-1}}\left\|A^{*}\left(Y^{-t}-X^{-t}\right) A+B^{*}\left(Y^{-t}-X^{-t}\right) B\right\|_{F} \\
& \leq \frac{1}{s \beta_{1}^{s-1}}\left(\|A\|_{2}^{2}+\|B\|_{2}^{2}\right)\left\|Y^{-t}-X^{-t}\right\|_{F} \\
& \leq \frac{1}{s \beta_{1}^{s-1}}\left(\lambda_{1}\left(A^{*} A\right)+\lambda_{1}\left(B^{*} B\right)\right) t \beta_{1}^{-(t+1)}\|Y-X\|_{F} \\
& =\frac{t}{s} \frac{\lambda_{1}\left(A^{*} A\right)+\lambda_{1}\left(B^{*} B\right)}{\beta_{1}^{s+t}}\|X-Y\|_{F} \\
& =\frac{t}{s}\left(\frac{\lambda_{n}(Q)}{\beta_{1}^{s}}-1\right)\|X-Y\|_{F} \\
& <\|X-Y\|_{F} .
\end{aligned}
$$

Thus, we know that the map $G(X)$ is a contraction map in $\varphi_{4}$. By Banach fixed point theorem, the map $G(X)$ has a unique fixed point in $\varphi_{4}$ and this shows that (2.48) has a unique HPD solution in $\varphi_{4}$.

Assume $X$ is the HPD solution of (2.48), then

$$
\begin{aligned}
\lambda_{1}^{s}(X)=\lambda_{1}\left(X^{s}\right) & =\lambda_{1}\left(Q-A^{*} X^{-t} A-B^{*} X^{-t} B\right) \\
& \leq \lambda_{1}(Q)-\frac{\lambda_{n}\left(A^{*} A\right)+\lambda_{n}\left(B^{*} B\right)}{\lambda_{1}^{t}(X)},
\end{aligned}
$$

that is, $\lambda_{1}^{s+t}(X)-\lambda_{1}(Q) \lambda_{1}^{t}(X)+\lambda_{n}\left(A^{*} A\right)+\lambda_{n}\left(B^{*} B\right) \leq 0$. So, $\alpha_{1} \leq \lambda_{1}(X) \leq \beta_{2}$, thus (2.48) has no HPD solution in $\varphi_{1}, \varphi_{5}$.

$$
\begin{aligned}
\lambda_{n}^{S}(X)=\lambda_{n}\left(X^{s}\right) & =\lambda_{n}\left(Q-A^{*} X^{-t} A-B^{*} X^{-t} B\right) \\
& \geq \lambda_{n}(Q)-\frac{\lambda_{1}\left(A^{*} A\right)+\lambda_{1}\left(B^{*} B\right)}{\lambda_{n}^{t}(X)},
\end{aligned}
$$

that is, $\lambda_{n}^{s+t}(X)-\lambda_{n}(Q) \lambda_{n}^{t}(X)+\lambda_{1}\left(A^{*} A\right)+\lambda_{1}\left(B^{*} B\right) \geq 0$. So, $\lambda_{n}(X) \leq \alpha_{2}$ or $\lambda_{n}(X) \geq \beta_{1}$, thus (2.48) has no HPD solution in $\varphi 3$.

This completes the proof. 


\section{Iterative Method for the Maximal HPD Solution}

In this section, we consider the iterative method for obtaining the maximal HPD solution $X_{L}$ of (1.1). We propose the following algorithm which avoids calculating matrix inversion in the process of iteration.

Algorithm 1.

Step 1. Input initial matrices:

$$
\begin{aligned}
& X_{0}=r Q^{1 / s}, \\
& Y_{0}=\frac{\gamma+1}{2 \gamma} Q^{-1 / s},
\end{aligned}
$$

where $\gamma \in(\widehat{\alpha}, 1)$, and $\widehat{\alpha}$ is defined in Theorem 2.11.

Step 2. For $k=0,1,2, \ldots$, compute

$$
\begin{aligned}
Y_{k+1} & =Y_{k}\left(2 I-X_{k} Y_{k}\right), \\
X_{k+1} & =\left(Q-A^{*} Y_{k+1}^{t_{1}} A-B^{*} Y_{k+1}^{t_{2}} B\right)^{1 / s}
\end{aligned}
$$

Theorem 3.1. If (1.1) has an HPD solution, then it has the maximal one $X_{L}$. Moreover, to the sequences $X_{k}$ and $Y_{k}$ generated by Algorithm 1, one has

$$
X_{0}>X_{1}>X_{2}>\ldots, \lim _{k \rightarrow \infty} X_{k}=X_{L} ; \quad Y_{0}<Y_{1}<Y_{2}<\cdots, \lim _{k \rightarrow \infty} Y_{k}=X_{L}^{-1} .
$$

Proof. Since $X_{L}$ is an HPD solution of (1.1), by Theorem 2.11, we have $X_{L} \leq \widehat{\alpha} Q^{1 / s}$, thus

$$
X_{0}=\gamma Q^{1 / s}>\widehat{\alpha} Q^{1 / s} \geq X_{L}, \quad Y_{0}=\frac{\gamma+1}{2 \gamma} Q^{-1 / s}<\frac{1}{\gamma} Q^{-1 / s}<\frac{1}{\hat{\alpha}} Q^{-1 / s} \leq X_{L}^{-1} .
$$

By Lemmas 2.1 and 2.4, we have

$$
\begin{gathered}
Y_{1}=Y_{0}\left(2 I-X_{0} Y_{0}\right)=2 Y_{0}-Y_{0} X_{0} Y_{0} \leq X_{0}^{-1}<X_{L}^{-1}, \\
Y_{1}-Y_{0}=Y_{0}-Y_{0} X_{0} Y_{0}=Y_{0}\left(Y_{0}^{-1}-X_{0}\right) Y_{0}=\frac{1-\gamma^{2}}{4 \gamma} Q^{-1 / s}>0 .
\end{gathered}
$$

According to Lemma 2.1 and $Y_{1}<X_{L}^{-1}$, we have

$$
\begin{gathered}
X_{1}=\left(Q-A^{*} Y_{1}^{t_{1}} A-B^{*} Y_{1}^{t_{2}} B\right)^{1 / s}>\left(Q-A^{*} X_{L}^{-t_{1}} A-B^{*} X_{L}^{-t_{2}} B\right)^{1 / s}=X_{L}, \\
X_{1}^{s}-X_{0}^{s}=-A^{*}\left(Y_{1}^{t_{1}}-Y_{0}^{t_{1}}\right) A-B^{*}\left(Y_{1}^{t_{2}}-Y_{0}^{t_{2}}\right) B<0,
\end{gathered}
$$

that is, $X_{1}^{s}<X_{0}^{s}$, by Lemma 2.1 again, it follows that $X_{1}<X_{0}$.

Hence $X_{0}>X_{1}>X_{L}$, and $Y_{0}<Y_{1}<X_{L}^{-1}$. 
Assume that $X_{k-1}>X_{k}>X_{L}$, and $Y_{k-1}<Y_{k}<X_{L}^{-1}$, we will prove the inequalities $X_{k}>X_{k+1}>X_{L}$, and $Y_{k}<Y_{k+1}<X_{L}^{-1}$.

By Lemmas 2.1 and 2.4, we have

$$
\begin{gathered}
Y_{k+1}=2 Y_{k}-Y_{k} X_{k} Y_{k} \leq X_{k}^{-1}<X_{L}^{-1}, \\
X_{k+1}=\left(Q-A^{*} Y_{k+1}^{t_{1}} A-B^{*} Y_{k+1}^{t_{2}} B\right)^{1 / s}>\left(Q-A^{*} X_{L}^{-t_{1}} A-B^{*} X_{L}^{-t_{2}} B\right)^{1 / s}=X_{L} .
\end{gathered}
$$

Since $Y_{k} \leq X_{k-1}^{-1}<X_{k}^{-1}$, we have $Y_{k}^{-1}>X_{k}$, thus we have by Lemma 2.1 that

$$
\begin{gathered}
Y_{k+1}-Y_{k}=Y_{k}\left(Y_{k}^{-1}-X_{k}\right) Y_{k}>0, \\
X_{k+1}^{s}-X_{k}^{s}=-A^{*}\left(Y_{k+1}^{t_{1}}-Y_{k}^{t_{1}}\right) A-B^{*}\left(Y_{k+1}^{t_{2}}-Y_{k}^{t_{2}}\right) B<0,
\end{gathered}
$$

that is, $X_{k+1}^{s}<X_{k}^{s}$, by Lemma 2.1 again, it follows that $X_{k+1}<X_{k}$.

Hence we have by induction that

$$
X_{0}>X_{1}>X_{2}>\cdots>X_{k}>X_{L}, \quad Y_{0}<Y_{1}<Y_{2}<\cdots<Y_{k}<X_{L}^{-1}
$$

are true for all $k=0,1,2, \ldots$, and so $\lim _{k \rightarrow \infty} X_{k}$ and $\lim _{k \rightarrow \infty} Y_{k}$ exist. Suppose $\lim _{k \rightarrow \infty} X_{k}=\hat{X}$, $\lim _{k \rightarrow \infty} Y_{k}=\widehat{Y}$, taking the limit in the Algorithm 1 leads to $\widehat{Y}=\widehat{X}^{-1}$ and $\widehat{X}=\left(Q-A^{*} \widehat{X}^{-t_{1}} A-\right.$ $\left.B^{*} \hat{X}^{-t_{2}} B\right)^{1 / s}$. Therefore $\widehat{X}$ is an HPD solution of (1.1), thus $\hat{X} \leq X_{L}$. Moreover, as each $X_{k}>X_{L}$, so $\widehat{X} \geq X_{L}$, then $\widehat{X}=X_{L}$. The theorem is proved.

Theorem 3.2. If (1.1) has an HPD solution and after $k$ iterative steps of Algorithm 1, one has $\| I$ $X_{k} Y_{k} \|<\varepsilon$, then

$$
\left\|X_{k}^{s}+A^{*} X_{k}^{-t_{1}} A+B^{*} X_{k}^{-t_{2}} B-Q\right\| \leq \varepsilon \lambda_{n}^{-1}(\bar{M})\left(t_{1} \lambda_{1}^{\left(1-t_{1}\right) / s}(Q)\|A\|^{2}+t_{2} \lambda_{1}^{\left(1-t_{2}\right) / s}(Q)\|B\|^{2}\right),
$$

where $\bar{M}$ is defined by (2.13).

Proof. From the proof of Theorem 3.1, we have $Q^{-1 / s}<((\gamma+1) / 2 \gamma) Q^{-1 / s}<Y_{k}<X_{k}^{-1}<X_{L}^{-1}$ for all $k=1,2, \ldots$. Thus we have by Theorem 2.9 that $Q^{-1 / s}<Y_{k}<X_{k}^{-1}<\bar{M}^{-1}$. And this implies

$$
\lambda_{1}^{-1 / s}(Q) I<Y_{k}<X_{k}^{-1}<\lambda_{n}^{-1}(\bar{M}) I .
$$

Since

$$
\begin{aligned}
X_{k}^{s}+A^{*} X_{k}^{-t_{1}} A+B^{*} X_{k}^{-t_{2}} B-Q & =\left(Q-A^{*} Y_{k}^{t_{1}} A-B^{*} Y_{k}^{t_{2}} B\right)+A^{*} X_{k}^{-t_{1}} A+B^{*} X_{k}^{-t_{2}} B-Q \\
& =A^{*}\left(X_{k}^{-t_{1}}-Y_{k}^{t_{1}}\right) A+B^{*}\left(X_{k}^{-t_{2}}-Y_{k}^{t_{2}}\right) B,
\end{aligned}
$$


we have by Lemma 2.5 that

$$
\begin{aligned}
\left\|X_{k}^{s}+A^{*} X_{k}^{-t_{1}} A+B^{*} X_{k}^{-t_{1}} B-Q\right\| & =\left\|A^{*}\left(X_{k}^{-t_{1}}-Y_{k}^{t_{1}}\right) A+B^{*}\left(X_{k}^{-t_{2}}-Y_{k}^{t_{2}}\right) B\right\| \\
& \leq\|A\|^{2}\left\|X_{k}^{-t_{1}}-Y_{k}^{t_{1}}\right\|+\|B\|^{2}\left\|X_{k}^{-t_{2}}-Y_{k}^{t_{2}}\right\| \\
& \leq\left(t_{1} \lambda_{1}^{-\left(t_{1}-1\right) / s}(Q)\|A\|^{2}+t_{2} \lambda_{1}^{-\left(t_{2}-1\right) / s}(Q)\|B\|^{2}\right)\left\|X_{k}^{-1}-Y_{k}\right\| \\
& \leq\left(t_{1} \lambda_{1}^{\left(1-t_{1}\right) / s}(Q)\|A\|^{2}+t_{2} \lambda_{1}^{\left(1-t_{2}\right) / s}(Q)\|B\|^{2}\right)\left\|X_{k}^{-1}\right\|\left\|I-X_{k} Y_{k}\right\| \\
& \leq \varepsilon \lambda_{n}^{-1}(\bar{M})\left(t_{1} \lambda_{1}^{\left(1-t_{1}\right) / s}(Q)\|A\|^{2}+t_{2} \lambda_{1}^{\left(1-t_{2}\right) / s}(Q)\|B\|^{2}\right) .
\end{aligned}
$$

\section{Numerical Example}

In this section, we give a numerical example to illustrate the efficiency of the proposed algorithm. All the tests are performed by MATLAB 7.0 with machine precision around $10^{-16}$. We stop the practical iteration when the residual $\left\|X_{k}^{s}+A^{*} X_{k}^{-t_{1}} A+B^{*} X_{k}^{-t_{2}} B-Q\right\|_{F} \leq 1.0 e-010$.

Example 4.1. Let $s=5, t_{1}=0.2, t_{2}=0.5$, and

$$
\begin{aligned}
& A=\left(\begin{array}{llllll}
2 & 0 & 0 & 1 & 0 & 0 \\
1 & 2 & 0 & 0 & 1 & 0 \\
0 & 0 & 3 & 0 & 1 & 0 \\
1 & 0 & 0 & 2 & 0 & 1 \\
1 & 0 & 1 & 0 & 3 & 0 \\
0 & 1 & 0 & 0 & 1 & 2
\end{array}\right), \quad B=\left(\begin{array}{llllll}
2 & 1 & 6 & 0 & 5 & 7 \\
3 & 4 & 7 & 1 & 3 & 0 \\
0 & 9 & 2 & 4 & 7 & 8 \\
8 & 5 & 3 & 0 & 0 & 1 \\
2 & 5 & 0 & 2 & 1 & 7 \\
4 & 0 & 0 & 1 & 4 & 9
\end{array}\right), \\
& Q=\left(\begin{array}{llllllll}
105 & 66 & 58 & 15 & 41 & 73 \\
66 & 154 & 67 & 50 & 88 & 121 \\
58 & 67 & 109 & 15 & 71 & 61 \\
15 & 50 & 15 & 28 & 37 & 57 \\
41 & 88 & 71 & 37 & 113 & 136 \\
73 & 121 & 61 & 57 & 136 & 250
\end{array}\right) .
\end{aligned}
$$


By calculating, $\widehat{\alpha} \approx 0.8397136$, so we choose $\gamma=0.84$. By using Algorithm 1 and iterating 29 steps, we obtain the maximal HPD solution $X_{L}$ of (1.1) as follows:

$$
X_{L} \approx X_{29}=\left(\begin{array}{cccccc}
1.6657 & 0.1110 & 0.2391 & 0.0105 & 0.0566 & 0.2624 \\
0.1110 & 1.8337 & 0.2270 & 0.2870 & 0.2656 & 0.2483 \\
0.2391 & 0.2270 & 1.7238 & -0.0037 & 0.3058 & 0.0662 \\
0.0105 & 0.2870 & -0.0037 & 1.1501 & 0.1227 & 0.1739 \\
0.0566 & 0.2656 & 0.3058 & 0.1227 & 1.5140 & 0.4831 \\
0.2624 & 0.2483 & 0.0662 & 0.1739 & 0.4831 & 2.1751
\end{array}\right),
$$

with the residual $\left\|X_{29}^{5}+A^{*} X_{29}^{-0.2} A+B^{*} X_{29}^{-0.5} B-Q\right\|_{F}=9.9360 e-011$.

\section{Acknowledgments}

The authors express their thanks to the editor Professor Mohammad I. Younis and the anonymous referees who made much useful and detailed suggestions that helped them to correct some minor errors and improve the quality of the paper. This project was granted financial support from Natural Science Foundation of China (11071079), Natural Science Foundation of China (11001167), Natural Science Foundation of China (10901056), Natural Science Foundation of Zhejiang Province (Y6110043), and Shanghai Natural Science Foundation (09ZR1408700).

\section{References}

[1] J. Long, X. Hu, and L. Zhang, "On the Hermitian positive definite solution of the nonlinear matrix equation $X+A^{*} X^{-1} A+B^{*} X^{-1} B=I$," Bulletin of the Brazilian Mathematical Society, vol. 39, no. 3, pp. 371-386, 2008.

[2] W. N. Anderson, Jr., T. D. Morley, and G. E. Trapp, “Positive solutions to $X=A-B X^{-1} B^{*}$," Linear Algebra and its Applications, vol. 134, pp. 53-62, 1990.

[3] X. Chen and W. Li, "On the matrix equation $X+A^{*} X^{-1} A=P$ : solution and perturbation analysis," Mathematica Numerica Sinica, vol. 27, no. 3, pp. 303-310, 2005 (Chinese).

[4] J. C. Engwerda, A. C. M. Ran, and A. L. Rijkeboer, "Necessary and sufficient conditions for the existence of a positive definite solution of the matrix equation $X+A^{*} X^{-1} A=Q$, , Linear Algebra and its Applications, vol. 186, pp. 255-275, 1993.

[5] J. C. Engwerda, "On the existence of a positive definite solution of the matrix equation $X+A^{*} X^{-1} A=$ I," Linear Algebra and its Applications, vol. 194, pp. 91-108, 1993.

[6] V. I. Hasanov, "Positive definite solutions of the matrix equations $X \pm A^{*} X^{-q} A=Q$," Linear Algebra and its Applications, vol. 404, pp. 166-182, 2005.

[7] V. I. Hasanov and S. M. El-Sayed, "On the positive definite solutions of nonlinear matrix equation $X+A^{*} X^{-\delta} A=Q, "$ Linear Algebra and its Applications, vol. 412, no. 2-3, pp. 154-160, 2006.

[8] Z. Peng, S. M. El-Sayed, and X. Zhang, "Iterative methods for the extremal positive definite solution of the matrix equation $X+A^{*} X^{-\alpha} A=Q$," Journal of Computational and Applied Mathematics, vol. 200, no. 2, pp. 520-527, 2007.

[9] J. Cai and G. Chen, "Some investigation on Hermitian positive definite solutions of the matrix equation $X^{s}+A^{*} X^{-t} A=Q, "$ Linear Algebra and its Applications, vol. 430, no. 8-9, pp. 2448-2456, 2009.

[10] J. Cai and G. Chen, "On the Hermitian positive definite solutions of nonlinear matrix equation $X^{s}+$ $A^{*} X^{-t} A=Q, "$ Applied Mathematics and Computation, vol. 217, no. 1, pp. 117-123, 2010.

[11] M. Parodi, La Localisation des Valeurs Caractéristiques des Matrices et ses Applications, Traité de Physique Théorique et de Physique Mathématique, XII, Gauthier-Villars, Paris, France, 1959. 
[12] T. Furuta, "Operator inequalities associated with Holder-McCarthy and Kantorovich inequalities," Journal of Inequalities and Applications, vol. 2, no. 2, pp. 137-148, 1998.

[13] X. Duan and A. Liao, "On the existence of Hermitian positive definite solutions of the matrix equation $X^{s}+A^{*} X^{-t} A=Q$," Linear Algebra and its Applications, vol. 429, no. 4, pp. 673-687, 2008.

[14] X. Zhan, "Computing the extremal positive definite solutions of a matrix equation," SIAM Journal on Scientific Computing, vol. 17, no. 5, pp. 1167-1174, 1996.

[15] R. Bhatia, Matrix Analysis, vol. 169 of Graduate Texts in Mathematics, Springer, Berlin, Germany, 1997.

[16] J. Sun, Matrix Perturbation Analysis, Science Press, Beijing, China, 2001. 


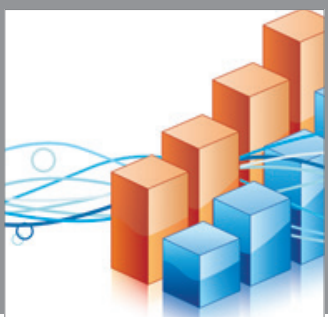

Advances in

Operations Research

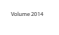

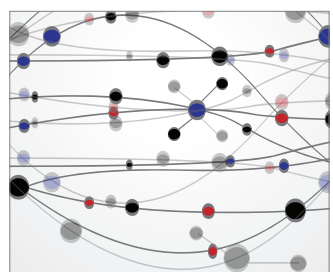

\section{The Scientific} World Journal
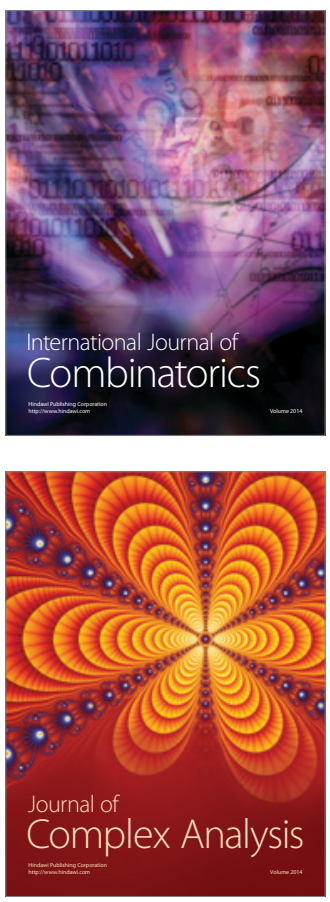

International Journal of

Mathematics and

Mathematical

Sciences
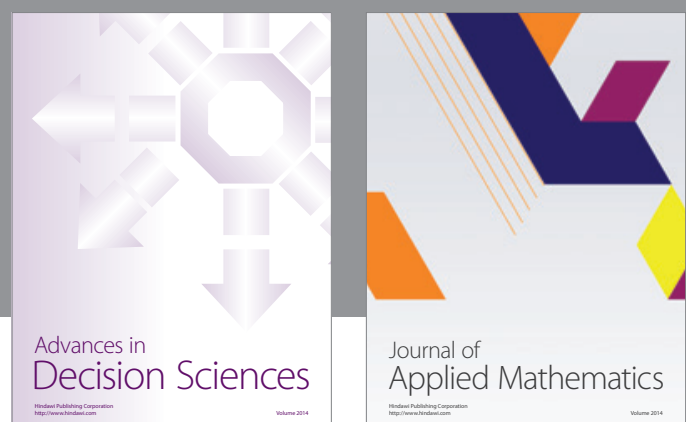

Journal of

Applied Mathematics
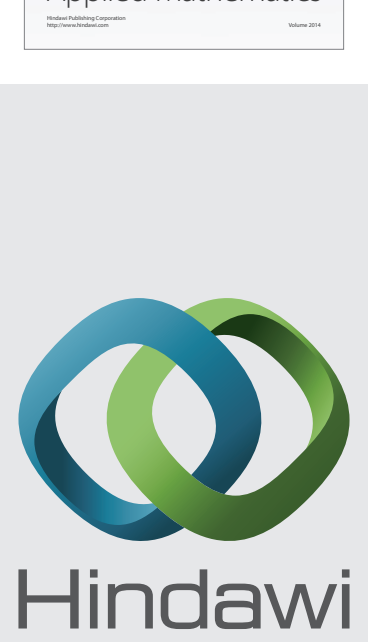

Submit your manuscripts at http://www.hindawi.com
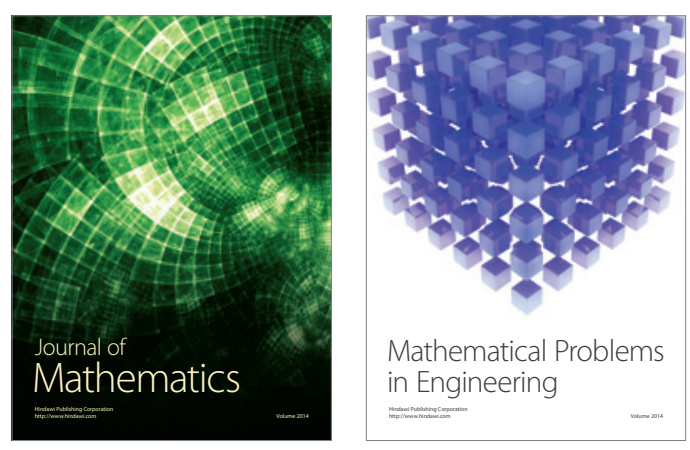

Mathematical Problems in Engineering
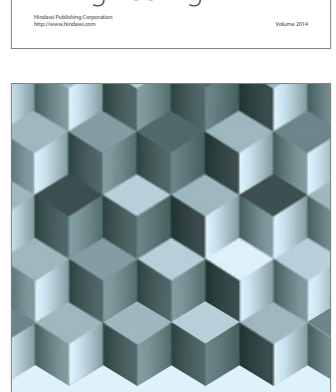

Journal of

Function Spaces
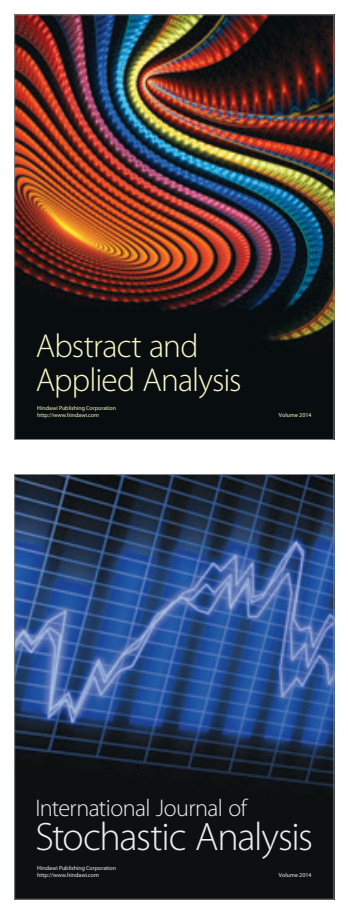

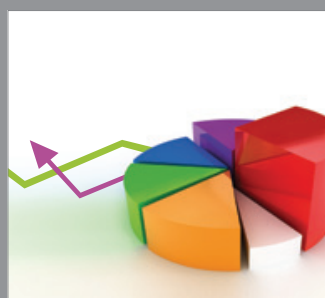

ournal of

Probability and Statistics

Promensencen
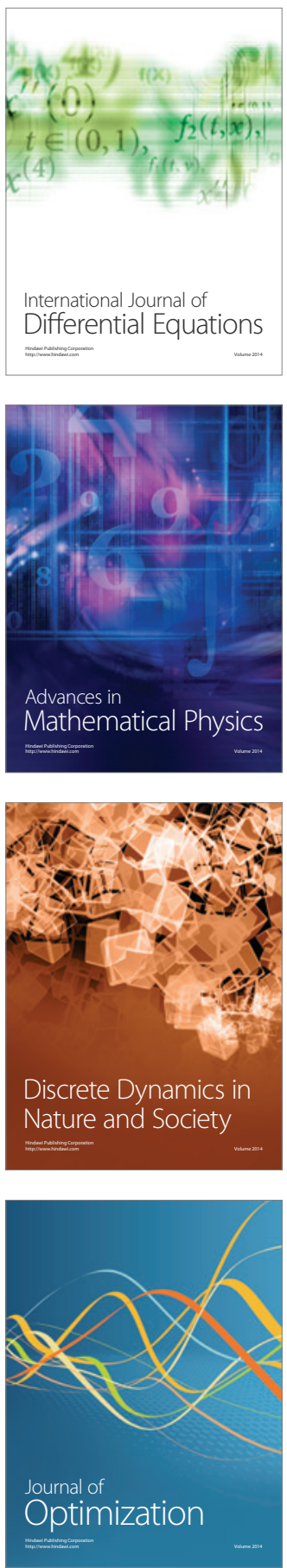